\title{
On the Ergodic Capacity of the Wideband MIMO Channel
}

\author{
Symeon Chatzinotas, Muhammad Ali Imran, Reza Hoshyar \\ Centre for Communication Systems Research \\ University of Surrey, United Kingdom, GU2 7XH \\ Email: \{S.Chatzinotas, M.Imran, R.Hoshyar\}@ surrey.ac.uk
}

\begin{abstract}
In the ergodic capacity literature, the majority of results preserve the assumption of a flat-fading Gaussian channel. However, the actual wireless channels in current communication systems are often wideband and therefore time dispersive and frequency selective. In this direction, we study the ergodic capacity of a wideband MIMO channel. Starting from a tapped delay line model for the time domain, the frequency domain model is derived by employing the Fourier transform. However, in both cases the resulting channel matrix is characterised by nonseparable correlation amongst the Gaussian blocks. The asymptotic eigenvalue probability density function and the channel capacity are calculated by specializing a theorem originating in operator-valued free probability. Finally, numerical results are presented to verify the validity of the approach and study the effect of the channel model parameters.
\end{abstract}

\section{INTRODUCTION}

Across the information-theoretic literature, it has been widely proven that multiple antennas are able to increase the link throughput by providing a multiplexing gain which scales with the number of antennas [1]. Similarly, in the context of multiuser channels, the sum-rate capacity of a MIMO multiple-access channel was investigated in [2], [3]. In both cases, it was shown that the capacity scales linearly with $\min \left(n_{R}, n_{T}\right)$, where $n_{R}$ and $n_{T}$ is the total number of receive and transmit antennas respectively. However, the majority of results focus on flat-fading channels, which is a valid assumption for narrowband channels. Nevertheless, current wireless technologies have adopted wideband channels, which are characterized by time dispersion and frequency selectivity. Time dispersive channels can be modelled by tapped delay line models and subsequently can be converted in the frequency domain by employing the Fourier transform. However, as shown in the channel modelling section this approach induces a non-separable correlation in the channel matrix. This kind of correlation is characterized "non-separable" in contradiction to the "separable" case, which can be modelled as the product of a receive correlation matrix, a Gaussian matrix and a transmit correlation matrix [4], [5], [6]. The capacity scaling of the wideband MIMO channel was also studied in [7] based on the principles of virtual representation. In our approach, we specialize a theorem originating in operatorvalued free probability in order to calculate the asymptotic eigenvalue probability density function of the channel matrix and consequently the ergodic channel capacity.

The remainder of this paper is organized as follows. Section
II defines the time- and frequency domain representation of the wideband MIMO channel. Subsequently, section III focuses on the analysis of the non-separable correlation model and section IV presents some numerical results in order to verify the validity of the approach and study the effect of the channel model parameters. Section V concludes the paper.

\section{Channel Model}

The current model considers a MIMO channel with $n_{T}$ transmit and $n_{R}$ receive antennas. The communication channel is wideband and thus it is characterized by time dispersion and frequency selectivity. Starting from a tapped delay line model for the time domain, the frequency domain model is derived by employing the Fourier transform. In this context, $N_{c}$ denotes the number of flat-faded narrowband subcarriers in the frequency domain and the number of frame symbols in the time domain. It should be noted that the main part of the analysis focuses on equal number of transmit and receive antennas $n_{T}=n_{R}$, while the case of $n_{T} \neq n_{R}$ is considered in subsection III-A3.

\section{A. Time-Domain Wideband Single-Link Channel}

Let us first consider a single-antenna link, which transmits in wideband mode. Due to the time dispersion, the received signal at a time index $m$ is given by:

$$
y[m]=\sum_{l=1}^{L} h_{l}^{t}[m] x[m-(l-1)]+n[m],
$$

where $L$ is a finite number of independent Rayleigh faded channel taps with independent complex circularly symmetric (c.c.s.) gains $h_{l}^{t}, \forall l=1 \ldots L, x[m]$ denotes the symbol transmitted at time index $m$ and $n$ represents the Additive White Gaussian Noise (AWGN). The variance $v_{l}$ of $h_{l}^{t}$ decays as $l \rightarrow L$ under the normalization

$$
\sum_{l=1}^{L} \mathbb{E}\left[h_{l}^{t}\left(h_{l}^{t}\right)^{\dagger}\right]=\sum_{l=1}^{L} v_{l}^{2}=1 .
$$

Assuming that $L$ is smaller than $N_{c}$, then the time-domain channel matrix $\mathbf{H}_{t}$ over a frame size $N_{c}$ can be written as a 
square matrix with dimensions $N_{c} \times N_{c}$ :

$$
\mathbf{H}_{t}=\left[\begin{array}{ccccccc}
h_{L}^{t} & h_{L-1}^{t} & \cdots & h_{1}^{t} & 0 & \cdots & 0 \\
0 & h_{L}^{t} & \cdots & h_{2}^{t} & h_{1}^{t} & \cdots & 0 \\
\vdots & \vdots & \cdots & \vdots & \vdots & \cdots & \vdots \\
h_{L-1}^{t} & h_{L-2}^{t} & \cdots & 0 & 0 & \cdots & h_{L}^{t}
\end{array}\right]
$$

It should be noted that the channel taps' gains are preserved for the duration of the frame. Furthermore, the matrix $\mathbf{H}_{t}$ appears as circulant, after employing a cyclic prefix on the transmitted symbols [8]. The channel model can be compactly expressed as:

$$
\mathbf{y}_{t}=\mathbf{H}_{t} \mathbf{x}_{t}+\mathbf{n}_{t}
$$

where $\mathbf{x}_{t}=\left[x[2-L] \ldots x\left[N_{c}-L+1\right]\right]^{T}$ and $\mathbf{y}_{t}=$ $\left[y[1] \ldots y\left[N_{c}\right]\right]^{T}$ are the transmitted and received symbols respectively and $\mathbf{n}_{t}$ is the AWGN vector. It is assumed that no input optimization is performed and thus the available transmit power $P$ is uniformly allocated across the $N_{c}$ transmitted frame symbols.

\section{B. Frequency-Domain Wideband Single-Link Channel}

Let us now consider a single antenna link, which transmits over $N_{c}$ flat-faded narrowband subcarriers with bandwidth $B_{c}=B / N_{c}$, where $B$ is the total communication bandwidth. The frequency-selective channel model can be compactly expressed as:

$$
\mathbf{y}_{f}=\mathbf{H}_{f} \mathbf{x}_{f}+\mathbf{n}_{f},
$$

where $\mathbf{x}_{f}$ and $\mathbf{y}_{f}$ are the transmitted and received subcarriers symbols respectively and $\mathbf{n}_{f}$ is the AWGN vector. The $N_{c} \times N_{c}$ frequency-domain channel matrix $\mathbf{H}_{f}$ is defined in accordance with the time-domain channel as follows

$$
\mathbf{H}_{f}=\left[\begin{array}{cccc}
h_{1}^{f} & 0 & \cdots & 0 \\
0 & h_{2}^{f} & \cdots & 0 \\
\vdots & \vdots & \ddots & \vdots \\
0 & 0 & \cdots & h_{N_{c}}^{f}
\end{array}\right]
$$

where the $i^{t h}$ diagonal entry is given by the Fourier transform:

$$
h_{i}^{f}=\sum_{l=1}^{L} h_{l}^{t} \exp \left(\frac{-\mathrm{j} 2 \pi(i-1)(l-1)}{N_{c}}\right) .
$$

As it can be seen, the subcarrier flat-fading coefficients $h_{i}^{f}$ are correlated due to (7). It is assumed that no input optimization is performed and thus the available power $P$ is uniformly allocated across the $N_{c}$ transmitted subcarrier symbols.

\section{Wideband MIMO Channel}

Considering $n_{R}=n_{T}$ multiple receive and transmit antennas, the wideband MIMO channel can be written in the form:

$$
\mathbf{Y}=\mathbf{H X}+\mathbf{Z},
$$

where the vector $\mathbf{Y}=\left[\begin{array}{lll}\mathbf{y}^{(1)} \ldots & \mathbf{y}^{\left(n_{R}\right)}\end{array}\right]^{T}$ with $\mathbf{y}^{(n)}=$

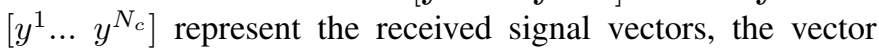

$\mathbf{X}=\left[\mathbf{x}^{(1)} \ldots \mathbf{x}^{\left(n_{T}\right)}\right]^{T}$ with $\mathbf{x}^{(n)}=\left[x^{1} \ldots x^{N_{c}}\right]$ represents transmitted signal vectors and the components of vector $\mathbf{Z}=\left[\begin{array}{lll}\mathbf{z}^{(1)} \ldots & \mathbf{z}^{\left(n_{R}\right)}\end{array}\right]^{T}$ with $\mathbf{z}^{(n)}=\left[\begin{array}{lll}z^{1} \ldots & z^{N_{c}}\end{array}\right]$ contain independent identically distributed (i.i.d) c.c.s. random variables representing AWGN. Considering that $N_{c}, L$ are preserved for all transmit-receive antenna pairs, the $n_{R} N_{c} \times n_{T} N_{c}$ channel matrix can be expressed as:

$$
\mathbf{H}=\left[\begin{array}{cccc}
\mathbf{H}_{1,1} & \mathbf{H}_{1,2} & \cdots & \mathbf{H}_{1, n_{T}} \\
\mathbf{H}_{2,1} & \mathbf{H}_{2,2} & \cdots & \vdots \\
\vdots & \vdots & \ddots & \vdots \\
\mathbf{H}_{n_{R}, 1} & \cdots & \cdots & \mathbf{H}_{n_{R}, n_{T}}
\end{array}\right]
$$

where $\mathbf{H}_{i, j}$ is the channel matrix of the link between the $i$ th receive antenna and the $j$ th transmit antenna. For the timedomain analysis $\mathbf{H}_{i, j}$ is considered to be an instance of $\mathbf{H}_{t}$, while for the frequency-domain analysis an instance of $\mathbf{H}_{f}$. As mentioned before, the available power $P$ is allocated uniformly across the $n_{T}$ transmit antennas and across the $N_{c}$ transmitted symbols and therefore $\mathbb{E}\left[\mathbf{x}_{t} \mathbf{x}_{t}^{\dagger}\right]=\mathbb{E}\left[\mathbf{x}_{f} \mathbf{x}_{f}^{\dagger}\right]=\frac{\gamma}{n_{T} N_{c}} \mathbf{I}$.

\section{CAPACITy ANALYSis}

In this section, we study the ergodic capacity normalized by the number of receive antennas $n_{R}$ and the frame size $N_{c}$. The normalized capacity assuming uniform power allocation across the transmission dimensions and $n_{R}=n_{T}=n$ is given by [9]:

$$
\begin{aligned}
& C_{\text {opt }}=\lim _{n \rightarrow \infty} \frac{1}{n_{R} N_{c}} \mathcal{I}(\mathbf{x} ; \mathbf{y} \mid \mathbf{H}) \\
& =\lim _{n \rightarrow \infty} \frac{1}{n_{R} N_{c}} \mathbb{E}\left[\log \operatorname{det}\left(\mathbf{I}+\frac{\gamma}{n_{T} N_{c}} \mathbf{H H}^{\dagger}\right)\right] \\
& =\lim _{n \rightarrow \infty} \mathbb{E}\left[\frac{1}{n_{R} N_{c}} \sum_{i=1}^{n_{R} N_{c}} \log \left(1+2 \gamma \lambda_{i}\left(\frac{1}{2 n_{T} N_{c}} \mathbf{H H}^{\dagger}\right)\right)\right] \\
& =\int_{0}^{\infty} \log (1+2 \gamma x) f_{\frac{1}{2 n_{T} N_{c}} \mathbf{H H}^{\dagger}}^{\infty}(x) d x
\end{aligned}
$$

where $\mathbb{E}$ denotes expectation, $\lambda_{i}(\mathbf{X})$ denotes the eigenvalues of matrix $\mathbf{X}$ and $f_{\mathbf{X}}^{\infty}$ denotes the asymptotic eigenvalue probability density function (a.e.p.d.f.) of matrix $\mathbf{X}$. Thus, it is requisite to calculate the a.e.p.d.f. of matrix $\frac{1}{2 n_{T} N_{c}} \mathbf{H} \mathbf{H}^{\dagger}$, which can be obtained by determining the imaginary part of the Cauchy transform $G$ for real arguments [9]:

$$
f_{\frac{1}{2 n_{T} N_{c}}}^{\infty} \mathbf{H H}^{\dagger}(x)=\lim _{y \rightarrow 0^{+}} \frac{1}{\pi} \mathfrak{I}\left\{G \frac{1}{2 n_{T} N_{c}} \mathbf{H H}^{\dagger}(x+\mathrm{j} y)\right\} .
$$

\section{A. Time-Domain Wideband MIMO Channel}

By reordering the rows and columns in the time domain, the $\mathbf{H}$ matrix can be written as:

$$
\mathbf{H}=\left[\begin{array}{ccccccc}
\mathbf{H}_{1}^{t} & \mathbf{H}_{2}^{t} & \cdots & \mathbf{H}_{L}^{t} & \mathbf{0} & \cdots & \mathbf{0} \\
\mathbf{0} & \mathbf{H}_{1}^{t} & \cdots & \mathbf{H}_{L-1}^{t} & \mathbf{H}_{L}^{t} & \cdots & \mathbf{0} \\
\vdots & \vdots & \cdots & \vdots & \vdots & \cdots & \vdots \\
\mathbf{H}_{2}^{t} & \mathbf{H}_{3}^{t} & \cdots & \mathbf{0} & \mathbf{0} & \cdots & \mathbf{H}_{1}^{t}
\end{array}\right]
$$


where $\mathbf{H}_{l}^{t}, \forall l=1 \ldots L$ are $n_{R} \times n_{T}$ channel matrices, describing the MIMO fading coefficients for each symbol of the frame. The entries in each matrix $\mathbf{H}_{l}^{t}$ are independent and jointly Gaussian, while correlation appears between the square blocks of matrix $\mathbf{H}$ in the form of repetition. This kind of correlation is non-separable, since it cannot be expressed as a product of a correlation matrix and a Gaussian matrix, as in [10].

1) Non-Separable Correlation: According to [11, Th.1], if $\mathbf{H}$ is a square $n_{R} N_{c} \times n_{T} N_{c}$ block matrix containing correlated $n_{R} \times n_{T}$ Gaussian blocks, the Cauchy transform of the a.e.p.d.f. $\left(n_{R}, n_{T} \rightarrow \infty\right)$ for $\frac{1}{2 n_{T} N_{c}} \mathbf{H H}^{\dagger}$ can be calculated as follows:

$$
G_{\frac{1}{2 n_{T} N_{c}}} \mathbf{H H}^{\dagger}(z)=\frac{1}{N_{c}} \operatorname{Tr}\left(\mathcal{G}_{1}(z)\right),
$$

where $\mathcal{G}_{1}(z)$ is the upper left block of

$$
\mathcal{H}(z)=\left[\begin{array}{cc}
\mathcal{G}_{1}(z) & \mathbf{0} \\
\mathbf{0} & \mathcal{G}_{2}(z)
\end{array}\right]
$$

and $\mathcal{H}(z)$ satisfies the following equation $\forall z \in \mathbb{C}^{+}$

$$
z \mathcal{H}(z)=\mathbf{I}+z \eta(\mathcal{H}(z)) \cdot \mathcal{H}(z)
$$

where $\eta$ is the mapping

$$
\eta:\left[\begin{array}{ll}
\mathbf{D}_{1} & \mathbf{D}_{3} \\
\mathbf{D}_{4} & \mathbf{D}_{2}
\end{array}\right] \longmapsto\left[\begin{array}{cc}
\eta_{1}\left(\mathbf{D}_{2}\right) & \mathbf{0} \\
\mathbf{0} & \eta_{2}\left(\mathbf{D}_{1}\right)
\end{array}\right]
$$

with

$$
\begin{aligned}
{\left[\eta_{1}(\mathbf{D})\right]_{i, j} } & :=\frac{1}{2 N_{c}} \sum_{k, l=1}^{N_{c}} \tau(i, k ; j, l) \cdot[\mathbf{D}]_{k, l} \\
{\left[\eta_{2}(\mathbf{D})\right]_{k, l} } & :=\frac{1}{2 N_{c}} \sum_{i, j=1}^{N_{c}} \tau(i, k ; j, l) \cdot[\mathbf{D}]_{j, i} .
\end{aligned}
$$

The real-valued function $\tau$ depends on the cross correlation between identically-indexed elements of the matrix blocks and is defined as:

$$
\mathbb{E}\left[h_{p q}^{(i, k)}\left(h_{p q}^{(j, l)}\right)^{\dagger}\right]=\frac{1}{2 n_{T} N_{c}} \cdot \tau(i, k ; j, l)
$$

where $h_{p q}^{(i, k)}$ is the $(p, q)$ th element of the $(i, k)$ th block.

In this direction, we observe that the block cross-correlation function for the time-domain wideband MIMO channel is given by:

$$
\begin{aligned}
& \tau\left(i, j ; k, \bmod \left(j+k-i-1, N_{c}\right)+1\right) \\
& = \begin{cases}1 \quad & \text { if } 1 \leq i, k \leq N_{c} \\
& i \leq j \leq i+L-1 \\
& i+L-1 \leq N_{c} \\
1 & \text { if } 1 \leq i, k \leq N_{c} \\
& i \leq j \leq K \\
& 1 \leq j \leq i+L-1-K \\
& i+L-1>N_{c} \\
0 & \text { elsewhere }\end{cases}
\end{aligned}
$$

2) Iterative Mapping Solution: Equation (15) for a specific $z$ running along the real axis can be solved by iterating over the mapping [12]:

$$
\mathcal{W} \longmapsto \mathcal{M}_{z}(\mathcal{W})=\frac{1}{2}\left(\mathcal{W}+(-\mathrm{j} z \mathbf{I}+z \eta(\mathcal{W}))^{-1}\right),
$$

where $\mathcal{H}=-\mathrm{j} \mathcal{W}$. It should be noted that in some cases a simpler set of equations can be derived by observing the effect of mapping $\eta$. For example, in [11] the fact that $\eta$ maps diagonal matrices to diagonal matrices reduces the problem to a set of quadratic equations. The same applies to the frequency-domain case (c.f. III-B), whereas in the timedomain the structure is more complex, since there are offdiagonal zero-values as well.

3) Rectangular Gaussian Blocks, $n_{R} \neq n_{T}$ : So far we have considered an equal number of receive and transmit antennas. In case that $n_{R} \neq n_{T}$ and more specifically the ratio of transmit and receive antennas is $\alpha=n_{T} / n_{R}$, the Cauchy transform of the a.e.p.d.f. $\left(n_{R}, n_{T} \rightarrow \infty\right)$ for $\frac{1}{2 n_{T} N_{c}} \mathbf{H} \mathbf{H}^{\dagger}$ can be calculated as follows [11]:

$$
G_{\frac{1}{2 n^{N} N_{c}}} \mathbf{H H}^{\dagger}(z)=\frac{1}{4 N_{c}}(a+1) \operatorname{Tr}(\mathcal{H}(z))-\frac{(\alpha-1)}{2 z} .
$$

\section{B. Frequency-Domain Wideband MIMO Channel}

By reordering the rows and columns in the frequency domain, the $n_{R} N_{c} \times n_{T} N_{c} \mathbf{H}$ matrix can be written as:

$$
\mathbf{H}=\left[\begin{array}{cccc}
\mathbf{H}_{1}^{f} & \mathbf{0} & \cdots & \mathbf{0} \\
\mathbf{0} & \mathbf{H}_{2}^{f} & \cdots & \mathbf{0} \\
\vdots & \vdots & \ddots & \vdots \\
\mathbf{0} & \mathbf{0} & \cdots & \mathbf{H}_{N_{c}}^{f}
\end{array}\right]
$$

where $\mathbf{H}_{i}^{f}, \forall i=1 \ldots N_{c}$ are $n_{R} \times n_{T}$ channel matrices, describing the MIMO flat-fading coefficients for each frequency subcarrier. The entries in each matrix $\mathbf{H}_{l}^{f}$ are independent and jointly Gaussian, while correlation appears between the blocks of matrix $\mathbf{H}$ according to Equation (7). This kind of correlation is also non-separable. 
In the case of the frequency-domain Wideband MIMO channel the block cross-correlation function is given by:

$$
\tau(i, i ; j, j)=\left\{\begin{array}{ll}
\mathbb{E}\left[w_{i} w_{j}\right] & \text { for } 1 \leq i, j \leq N_{c} \\
0 & \text { elsewhere }
\end{array},\right.
$$

where $w_{i}$ is the $i$ th element of the $N_{c} \times 1$ vector

$$
\mathbf{w}=\operatorname{diag}\left(\mathbf{H}_{f_{(i, j)}}\right)=\mathbf{F h}_{t_{(i, j)}}
$$

and $\mathbf{F}$ is a $N_{c} \times N_{c}$ Fourier matrix, $\mathbf{H}_{f_{(i, j)}}$ is the frequency domain channel matrix and $\mathbf{h}_{t_{(i, j)}}=\left[\begin{array}{llll}h_{1}^{t} & \ldots h_{L}^{t} & 0 \ldots 0\end{array}\right]^{T}$ contains the channel tap gains for the link between the $i$ th receive antenna and the $j$ th transmit antenna. Using Equation (7), the block cross correlation function can be calculated as:

$$
\mathbb{E}\left[w_{i} w_{j}\right]=\sum_{l=1}^{L} v_{l}^{2} \exp \left(\frac{-\mathrm{j} 2 \pi(i+j-2)(l-1)}{N_{c}}\right)
$$

This channel modelling may result in complex values of $\tau$, which cannot be handled by [11, Th.1]. However, if the employed channel model produces real subcarrier cross correlation coefficients, the a.e.p.d.f. can be calculated in the frequency-domain using the Theorem in III-A1 and Equation (24).

Alternatively, the special parallel structure of the frequencydomain wideband MIMO channel can be exploited to simplify the capacity calculation. In this direction, Equation (10) can be written as:

$$
\begin{aligned}
& C_{\text {opt }}=\lim _{n \rightarrow \infty} \frac{1}{n_{R} N_{c}} \mathbb{E}\left[\log \operatorname{det}\left(\mathbf{I}+\frac{\gamma}{n_{T} N_{c}} \mathbf{H H}^{\dagger}\right)\right] \\
& =\lim _{n \rightarrow \infty} \frac{1}{n_{R} N_{c}} \sum_{i=1}^{N_{c}} \mathbb{E}\left[\log \operatorname{det}\left(\mathbf{I}+\frac{\gamma}{n_{T} N_{c}} \mathbf{H}_{i}^{f}\left(\mathbf{H}_{i}^{f}\right)^{\dagger}\right)\right] .
\end{aligned}
$$

As a result, the block-correlation effect is removed and the wideband ergodic capacity can be calculated as the sum of the well-studied capacities of the uncorrelated narrowband MIMO subcarriers.

\section{NumericAl Results}

This section is dedicated on comparing the analytical results with Monte Carlo simulations and evaluating the effect of channel model parameters, such as frame size and tap delay line power profile. The analytical results can be acquired by employing the appropriate block cross-correlation function $\tau$ and numerically integrating over the resulting a.e.p.d.f. On the other hand, Monte Carlo simulations have to be run and averaged over a large number of channel realizations. The advantage of the analytical approach is that it is independent of the MIMO system size and it can provide accurate capacity results even for small number of antennas.

For the a.e.p.d.f. results presented in this section, we have used a 4-tap delay line power profile defined as follows:

$$
v_{1}=0 d B, v_{2}=-2 d B, v_{3}=-10 d B, v_{4}=-20 d B
$$

For the capacity results, $\gamma$ was set to $30 d B$.

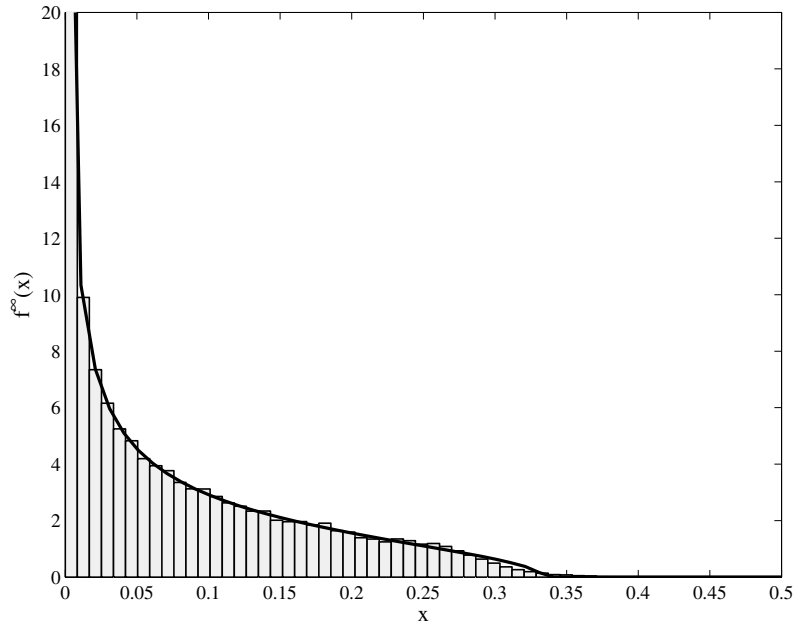

Fig. 1. Agreement between analytical (solid line) and simulated (bar plot) a.e.p.d.f. for $N_{c}=6$

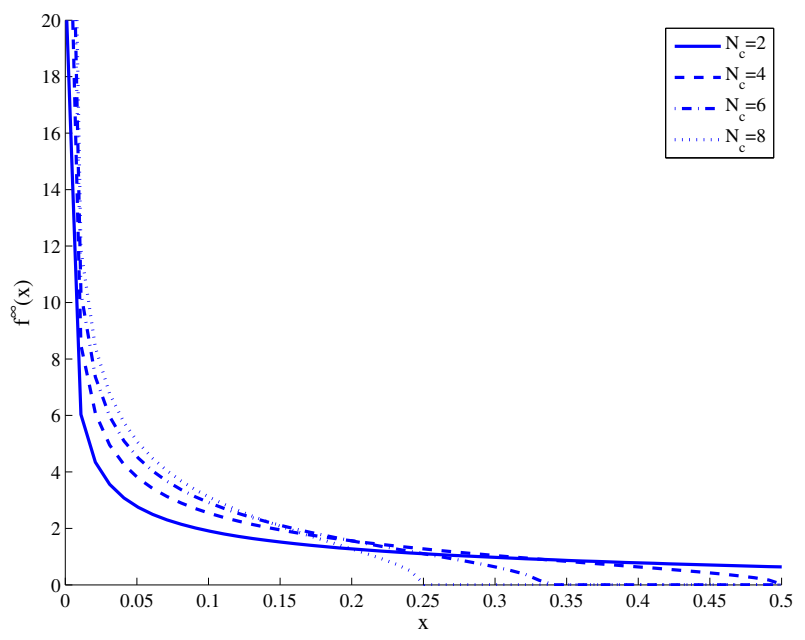

Fig. 2. The effect of frame size $N_{c}$ on the a.e.p.d.f. of the time-domain wideband MIMO channel

Figure 1 depicts the close agreement between the analytical and simulated e.p.d.f. The analytical curve was calculated using the block cross-correlation function in Equation (20), whereas for the simulated curve we used $n=10$ receive and transmit antennas over 1000 Monte Carlo iterations. Figure 2 illustrates the effect of frame size $N_{c}$ on the a.e.p.d.f. of the time-domain wideband MIMO channel, considering that the available power $P$ does not scale with the frame size $N_{c}$. In the same direction, Figure 3 shows the decay of the normalized capacity, which occurs by increasing $N_{c}$. It should be noted that if the transmitter power $P$ scales linearly with $N_{c}$, the produced capacity remains at the same level. In the previous numerical results, the tap delay power profile does not affect the capacity or the a.e.p.d.f. due to the normalization in Equation (2). In order to study this effect, we employ the tap delay power profile in Equation (28) without normalizing the power levels. In this context, Figure 4 depicts the normalized capacity versus the number of considered taps $L$. As expected, 


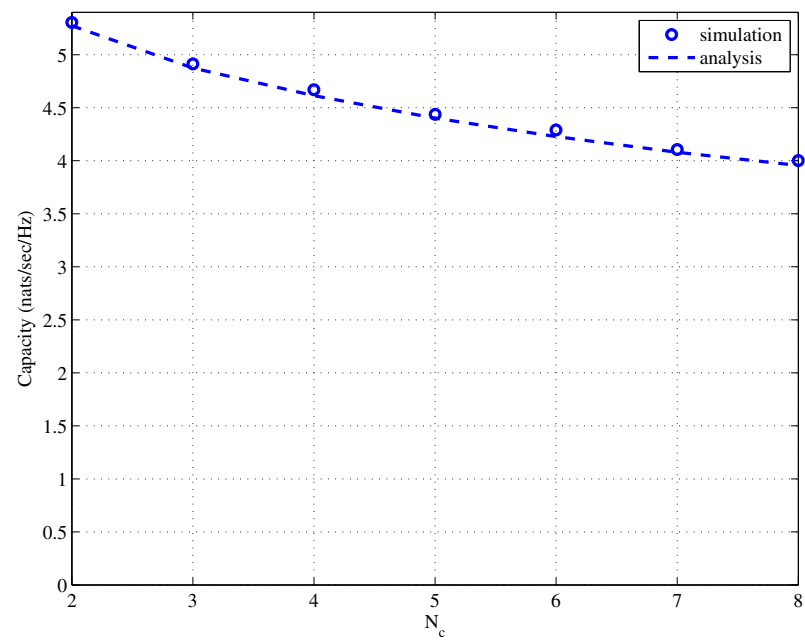

Fig. 3. Normalized Capacity in nats/sec/Hz vs the frame size $N_{c}$

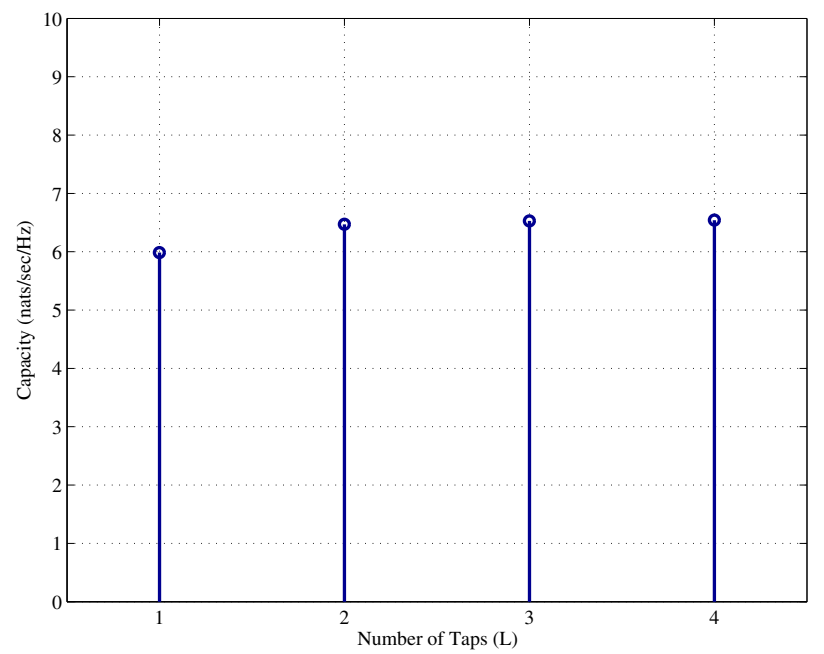

Fig. 4. Increase of normalized capacity (nats/sec/Hz) by increasing the considered number of taps $L$.

by including the high-power tap $v_{2}$ we can observe an increase of 0.5 nats $/ \mathrm{sec} / \mathrm{Hz}$, whereas taps $v_{3}$ and $v_{4}$ have a small effect on capacity due to their low power level.

\section{CONCLUSION}

In this paper, we have studied the ergodic capacity of the wideband MIMO channel in both time- and frequency domain. The main characteristic of the channel matrix in the wideband case is that non-separable correlation appears between the considered MIMO blocks and therefore the classic narrowband flat-fading approaches cannot be applied. In this direction, we have specialized the a.e.p.d.f. Theorem in [11] by defining appropriate block cross-correlation functions, which can be utilized in combination with an iterative mapping in order to calculate the ergodic channel capacity. Finally, a number of numerical results have been presented to verify the validity of the approach and study the effect of the channel model parameters.

\section{ACKNOWLEDGMENT}

The work reported in this paper has formed part of the "Fundamental Limits to Wireless Network Capacity" Elective Research Programme of the Virtual Centre of Excellence in Mobile \& Personal Communications, Mobile VCE, www.mobilevce.com. This research has been funded by the following Industrial Companies who are Members of Mobile VCE - BBC, BT, Huawei, Nokia, Nokia Siemens Networks, Nortel, Vodafone. Fully detailed technical reports on this research are available to staff from these Industrial Members of Mobile VCE. The authors would like to thank Prof. G. Caire and Prof. D. Tse for the useful discussions.

\section{REFERENCES}

[1] G. J. Foschini and M. J. Gans, "On limits of wireless communications in a fading environment when using multiple antennas," Wirel. Pers. Commun., vol. 6, no. 3, pp. 311-335, 1998.

[2] I. E. Telatar, "Capacity of multi-antenna Gaussian channels," European Transactions on Telecommunications, vol. 10, no. 6, pp. 585-595, Nov 1999.

[3] D. Aktas, M. Bacha, J. Evans, and S. Hanly, "Scaling results on the sum capacity of cellular networks with MIMO links," Information Theory, IEEE Transactions on, vol. 52, no. 7, pp. 3264-3274, July 2006.

[4] J. Kermoal, L. Schumacher, K. Pedersen, P. Mogensen, and F. Frederiksen, "A stochastic MIMO radio channel model with experimental validation," Selected Areas in Communications, IEEE Journal on, vol. 20, no. 6, pp. 1211-1226, Aug 2002.

[5] C.-N. Chuah, D. Tse, J. Kahn, and R. Valenzuela, "Capacity scaling in MIMO wireless systems under correlated fading," Information Theory, IEEE Transactions on, vol. 48, no. 3, pp. 637-650, Mar 2002.

[6] W. Hachem, O. Khorunzhiy, P. Loubaton, J. Najim, and L. Pastur, "A new approach for capacity analysis of large dimensional multi-antenna channels," 2006, submitted to IEEE Transactions on Information Theory.

[7] K. Liu, V. Raghavan, and A. Sayeed, "Capacity scaling and spectral efficiency in wide-band correlated MIMO channels," Information Theory, IEEE Transactions on, vol. 49, no. 10, pp. 2504-2526, Oct. 2003.

[8] A. Goldsmith, Wireless Communications. New York, NY, USA: Cambridge University Press, 2005.

[9] A. M. Tulino and S. Verdu, "Random matrix theory and wireless communications," Commun. Inf. Theory, vol. 1, no. 1, pp. 1-182, 2004.

[10] A. Skupch, D. Seethaler, and F. Hlawatsch, "Free probability based capacity calculation for MIMO channels with transmit or receive correlation," Wireless Networks, Communications and Mobile Computing, 2005 International Conference on, vol. 2, pp. 1041-1046 vol.2, 13-16 June 2005.

[11] R. Far, T. Oraby, W. Bryc, and R. Speicher, "On slow-fading MIMO systems with nonseparable correlation," Information Theory, IEEE Transactions on, vol. 54, no. 2, pp. 544-553, Feb. 2008.

[12] J. W. Helton, R. R. Far, and R. Speicher, "Operator-valued semicircular elements: Solving a quadratic matrix equation with positivity constraints," International Mathematics Research Notices, 2007. 